copiously produced. A narrow peak has been found in the invariant-mass distribution of the $(\psi \mathrm{K} \pi)$ system, at $5.3 \mathrm{GeV}$. It could be interpreted as a meson carrying the newly discovered b-quark (plus a light quark). This meson (usually denoted by $B$ ) has thus a new quantum number, associated to the b-quark (otherwise called beauty). Although the branching ratio for the weak decay of the B-meson into $\psi \mathrm{K} \pi$ is expected to be of the order of $1 \%$, this final state provides a good signature. If the peak is confirmed, it can be considered as the first evidence for a particle carrying the beauty quantum number.

From the study of the massive muon pair production with $\pi$ beams, the momentum distribution of the quarks inside the $\pi$ has been determined for the first time, in three experiments. The results are in qualitative agreement with the theoretical expectations.

In the theoretical sessions, several imaginative speculations were discussed at the Conference in connexion with the intriguing question of what is going to happen in the vast region of energies between the weak boson masses, of the order of $10^{2} \mathrm{GeV}$, and the Planck mass of $10^{19} \mathrm{GeV}$ where the gravitational interaction becomes relevant. This, however, is looking far to the future and there is already plenty to think about.

\section{C.A. Savoy}

Geneva University

\section{Electromagnetic Interactions of Hadrons}

edited by A. Donnachie and G. Shaw

University of Manchester, England

\section{in two volumes}

Examining the definitive evidence photoproduction experiments have provided for quark model ideas, and the similarities between high-energy electromagnetic interactions and hadron-induced reactions, these volumes focus on photoproduction and electroproduction in the resonance region and at medium energies, multiparticle production and inclusive reactions, deep inelastic scattering, and electron-positron annihilation. Volumes in Nuclear Physics Monographs.

Volume 1: 458 pp., illus., $1978, \$ 59.40 / £ 31.19$ outside US

Volume 2: 590 pp., illus., $1978, \$ 69.00 / £ 36.23$ outside US

\section{New Frontiers in High-Energy Physics} edited by Arnold PerImutter and Linda F. Scott University of Miami, Florida

This volume surveys recent advances in high-energy physics stressing results in the study of strong interactions, and examining several areas that will prove especially significant in the coming years. Studies in the Natural Sciences, Volume 14. 680 pp., 1978, $\$ 71.40 / £ 37.49$ outside US

\section{Plenifinim}

227 West 17th Street, New York, N.Y. 10011 Black Arrow House, 2 Chandos Road London NW10 6NR, England

\title{
Quantum Electronics Division
}

The 1979 General Business Meeting for all members of the Quantum Electronics Division will be held at Poggio Imperiale, Florence, on 3 September at 21.00 , to coincide with the Divisional Meeting on Lasers in Photomedicine and Photobiology, organised by Professor R. Pratesi, Laboratorio di Elettronica Quantistica, Via Panciatichi 56/

\section{EPS 5th GENERAL CONFERENCE}

\section{7-11 September, 1981}

The EPS 5th General Conference will be held in Istanbul in collaboration with the Turkish Physical Society. Chairman of the International Programme Committee is E. Matthias of Berlin (West) and of the Local Organizing Committee, E. Inönü of the Bogaziçi University, Istanbul.
30, I- 50127 Florence, tel. (55) 416128

The Quantum Electronics Division will be acting as an Advisory Programme Committee for the International Quantum Electronics Conference in Boston 1980. The Chairman (Professor S.D. Smith, Department of Physics, Heriot-Watt University, Riccarton, Currie, Edinburgh EH14 4AS, UK, telephone (0) 31-449 5542) would like to hear about any important work, worthy of inclusion.

The Quantum Electronics Division will be organising the First European Conference on Optical Systems and Applications in Utrecht from 23 to 25 September 1980. The chairman is Dr B. Bölger, Philips Research Laboratories, NL - 4500 Eindhoven, telephone (040) 742179 .
EPS Dlvisions, Sections and Group

Astronomy and Astrophysics Division Solar Section

Atomic Physics Division

Atomic Spectroscopy Section

Chemical Physics

Electronic and Atomic Collisions

Molecular Physics

Computational Physics Group

Condensed Matter Division

Low Temperature Section

Macromolecular Physics

Magnetism

Metals

Semiconductors and Insulators

Surface and Interface

High Energy \& Particle Physics Division

Nuclear Physics Division

Plasma Physics Division

Quantum Electronics Division
Europhysics News is the official journal of the European Physical Society that comprises $28 \mathrm{Na}$ tional Societies. Academies and Groups, over tional Societies, Academies and Groups, over
3000 Individual Ordinary Members and 30 Asso3000 Individual Ordinary Members and 30 Asso-
ciate Members. Governing bodies of EPS are the General Meeting, Council and an elected Executive Committee responsible for detailed policy EPS promotes the collaboration of physicists EPS promotes the collaboration of physicists throughout Europe and encourages all aspects of international exchange in physics. EPS awards scholarships for research and studies in different countries. EPS publishes, in addition to Europhys ics News, Europhysics Conference Abstracts, Europhysics Education News and the Proceedings of its General Conferences. Individual Ordinary Members receive Europhysics News (subscription (or non for non-Memb tes on many publications and pay reduced fees at conferences. Application for membership is made through the permanent Secretariat which is Jocated in Geneva. Annual subscription for members of a National Society is $28 \mathrm{Sw} . \mathrm{Fr}$.
Editor: E.N. Shaw

Meetings Compilation: W.S. Newman

Editorial Board

K. Appert, G.J. Béné, G.R. Macleod,

A. Maeder, J. Muller, D. Pohl

Editorial and Advertising Office at the EPS Secretariat.

Address: EUROPEAN PHYSICAL SOCIETY P.O. BoX 69, CH-1213 Petit-Lancy 2 Switzerland

Telephone: Geneva (22) 931130

Telex : 23455 alarm ch

Cables: europhys genève

Printed by : Ed. Cherix et Filanosa SA CH-1260 Nyon, Switzerland 